$$
\text { CONF. } 960401-45
$$

\title{
FORMATION AND PROPERTIES OF POROUS GaAs
}

P. SCHMUKI, D.J. LOCKWOOD, J.W. FRASER, M.J. GRAHAM, H.S. ISAACS* Institute for Microstructural Sciences, National Research Council of Canada, Ottawa, Ontario, Canada, K1A OR6

*Materials Science Division, Department of Applied Science, Brookhaven National Lab., Upton, NY 11973, USA

\section{ABSTRACT}

Porous structures on n-type GaAs $(100)$ can be grown electrochemically in chloridecontaining solutions. Crystallographic etching of the sample is a precursor stage of the attack. Polarization curves reveal the existence of a critical onset potential for pore formation (PFP). The PFP is strongly dependent on the doping level of the sample and the presence of surface defects. Good agreement between the PFP and the breakdown voltage of the space charge layer is found. Surface analytical investigations by EDX, AES and XPS show that the porous structure consists mainly of GaAs and that anion uptake in the structure can only be observed after attack has been initiated. Photoluminescence measurements reveal - under certain conditions - visible light emission from the porous structure.

\section{INTRODUCTION}

Electrochemical pore formation on semiconductors has recently received much attention. This is mainly the result of the remarkable light emitting properties of porous silicon, e.g., Refs. [1, 2]. For other semiconductor materials such as III-V compounds, despite their applications in optoelectronic devices, very little is known about the conditions to trigger localized dissolution, pore formation and subsequently the properties of the porous material. Pore formation has been reported for InP in $\mathrm{HCl}[3]$ and $\mathrm{GaP}$ in $\mathrm{H}_{2} \mathrm{SO}_{4}$ [4] electrolytes. According to the work of Tromans et. al. crystallographic etch pits can be triggered electrochemically on GaAs in neutral solutions $\left(\mathrm{NaCl}\right.$ and $\left.\mathrm{NaNO}_{3}\right)$ [5] . Recently the formation of porous $\mathrm{n}$ - and p-type $\mathrm{GaAs}$ was reported $[6,7]$. The present investigation extends the previous work by studying the influence of various parameters on the pore formation process on n-type GaAs. The aim of the work is to elucidate the electrochemical and chemical conditions under which local dissolution and pore formation occurs, to discuss the factors controlling this process, and to investigate the optical properties of porous GaAs.

\section{EXPERIMENTAL}

The materials used were n-type GaAs (100) wafers doped with $2.5 \cdot 10^{17} \mathrm{~cm}^{-3} \mathrm{Si}$ (if not denoted otherwise) and also $3.3 \cdot 10^{18} \mathrm{~cm}^{-3} \mathrm{Si}$ in expenments studying the influence of the doping concentration. Prior to each experiment the samples were degreased by successively sonicating in acetone, isopropanol and methanol and rinsing with distilled water. A subsequent etch in $1 \% \mathrm{HF}$ for 1 min removed the air formed oxide film [8]. Back contact to the $\mathrm{GaAs}$ was established by smearing In/Ga onto the samples. After cleaning, the samples were pressed against an O-ring in an electrochemical cell leaving $0.785 \mathrm{~cm}^{2}$ exposed to the electrolyte or alternatively by immersing the bottom half of a sample (held by a copper clip on a dot of $\mathrm{In} / \mathrm{Ga}$ ) into the electrolyte. Studies were carried out in $0.1 \mathrm{M} \mathrm{HCl}, 0.1 \mathrm{M} \mathrm{H}_{2} \mathrm{SO}_{4}$ and $0.3 \mathrm{M} \mathrm{NH} \mathrm{H}_{2} \mathrm{PO}_{4}$ buffer ( $\mathrm{pH} 4.4$ ), electrolytes. When desired, $\mathrm{NaCl}$ was added to the electrolytes to establish a predetermined $\mathrm{Cl}^{-}$- concentration. All solutions were prepared from analytical grade chemicals and deionized water.

The electrochemical cell consisted of a conventional 3-electrode configuration with a platinum gauze as a counter electrode and a Haber-Luggin capillary with a saturated calomel electrode (SCE) as a reference electrode. For polarization curves and potential step experiments, an EG\&G model 173 potentiostat was used. The polarization curves were carried out at a rate of $10 \mathrm{mV}$ every $5 \mathrm{sec}$. Unless otherwise stated, all electrochemical experiments were carried out in the dark. 
SEM micrographs were acquired using a JEOL 840A SEM equipped with a Link AN10000 EDX and a Digiscan Image acquisition archiving system. Energy Dispersive $X$-ray analysis (EDX) was performed semi-quantitatively in the spot mode, a difference in peak height representing a change in elemental concentration. Each feature was analyzed five times. The relative average elemental concentration was compared for each feature.

Measurements of Auger electron spectroscopy (AES) sputter depth profiles were performed in a Physical Electronics (PHI) model 650 system using a $1 \mathrm{keV}$ Argon ion beam and sputtering at $60^{\circ}$ off normal. The peaks analyzed were $\mathrm{Ga}(1070 \mathrm{eV}), \mathrm{As}(1228 \mathrm{eV}), \mathrm{O}(510 \mathrm{eV})$ and $\mathrm{Cl}(180$ $\mathrm{eV}$ ). All data were evaluated using PHI sensitivity factors except for Ga and As. In this case a clean $\mathrm{GaAs}$ sample was sputtered and the sensitivity factors adjusted to $S_{A s}=0.16$ and $S_{G a}=$ 0.089 which resulted in a $1: 1$ ratio of the atomic concentrations.

XPS spectra were acquired using a Perkin Elmer PHI 5500 system with a monochromated Al $K_{\alpha}$ source. The details of the analysis are given in Ref. [8].

The room temperature photoluminescence (PL) properties were studied using $15 \mathrm{~mW}$ of $457.9 \mathrm{~nm}$ argon laser light as the excitation source, a Spex 14018 double spectrometer; and a cooled RCA 31034A GaAs photomultiplier detector.

\section{RESULTS AND DISCUSSION}

\section{Electrochemistry}

Figure 1 shows polarization curves for $n-\mathrm{GaAs}$ in $0.1 \mathrm{M} \mathrm{H}_{2} \mathrm{SO}_{4}$ and $0.1 \mathrm{M} \mathrm{HCl}$ (Fig. 1a) as well as in $0.3 \mathrm{M} \mathrm{NH} \mathrm{H}_{2} \mathrm{PO}_{4}$ buffer solution with different chloride concentrations (Fig $1 \mathrm{~b}$ ). In the $\mathrm{H}_{2} \mathrm{SO}_{4}$ solution, the current remains low over a wide potential range. The presence of chloride, however, causes a significant change in the polarization behaviour. The curves show a plateau range followed by a distinct current increase at a certain potential value. At this potential the formation of dark spots on the sample can be observed visually. SEM micrographs reveal that pore formation takes place at this potential. Hence this potential value is assigned as the pore formation potential (PFP). Polarization of the sample for longer times above the PFP leads to complete coverage by a porous layer. The polarization behavior in phosphate-buffer is very similar to that in acidic solutions, i.e., pore formation at a distinct PFP in the presence of chloride ions and a wide potential range of low currents in the absence of chloride.

Figure 2 shows a comparison of polarization curves of $n-G a A s$ with the two doping levels in $\mathrm{HCl}$ solutions of different concentrations. It is clear that the PFP depends significantly on the doping concentration and depends to a much lesser extent on the $\mathrm{HCl}$ concentration. However samples exposed to a higher concentration of $\mathrm{HCl}$ generally exhibit a much more severe attack after polarization than samples treated in lower $\mathrm{HCl}$ concentrations. This indicates that the chloride concentration affects predominantly the pore growth process but only has a minor effect on the initiation process.

In repetitive experiments the magnitude of the current densities in the plateau range of the polarization curve show significant scatter. This can be attributed, as has been shown for $\mathrm{Si}$, to local defects in the surface, i.e., increased current flow at defects. A further indication that surface defects dominate the charge transfer processes is that the polarization curve of a scratched sample (included in Fig. 2a) shows significantly higher currents than a normal sample under the same conditions. For the scratched sample a significantly lower PFP is obtained. SEM investigations of the surface of a scratched sample polarized to 2.5V SCE - which is below the PFP of an unscratched sample - reveals that pore formation took place only in the region of the scratch. The rest of the surface remained intact. Hence dissolution occurred only locally at the scratch and the additional current flow can be attributed to this surface location, which means that the reaction proceeded with a very high local current density.

To explain these findings, the behavior of a n-type semiconductor/electrolyte junction under anodic bias is considered. In this case, depletion of majority carriers (electrons) takes place and a blocking space charge layer (Schottky barrier) is set up at the semiconductor/electrolyte interface, i.e., electron transfer with the surface is limited by tunneling through the depletion layer or by 


\section{DISCLAIMER}

Portions of this document may be illegible in electronic image products. Images are produced from the best available original document. 

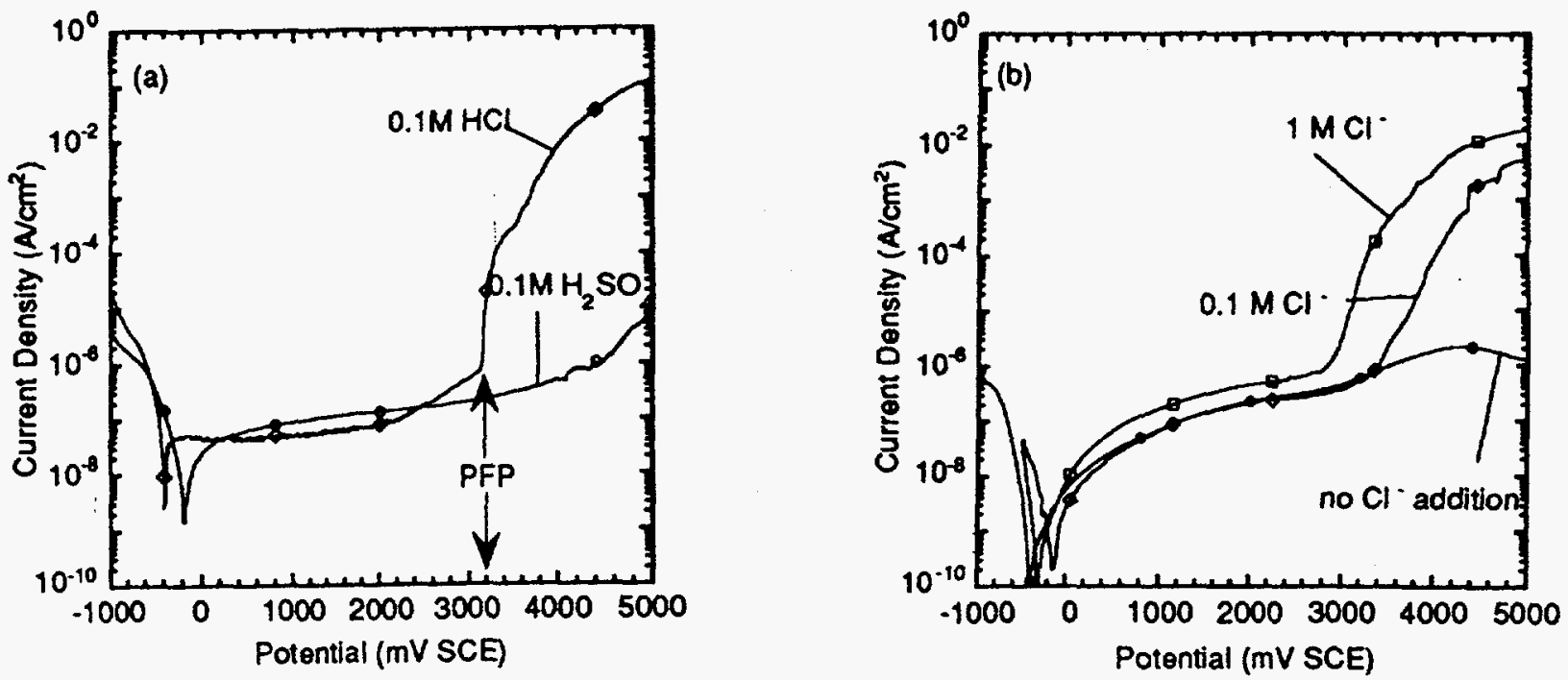

Fig. 1: Polarization curves of $n-G a A s(100)$ in: a) $0.1 \mathrm{M} \mathrm{H}_{2} \mathrm{SO}_{4}$ and $0.1 \mathrm{M} \mathrm{HCl}$. The pore formation potential (PFP) is indicated at $3.2 \mathrm{~V} \mathrm{SCE}$. b) in $0.3 \mathrm{M} \mathrm{NH}_{4} \mathrm{H}_{2} \mathrm{PO}_{4}$-buffer (pH 4.4) with different chloride ion concentrations. Sweep rate $5 \mathrm{mV} / \mathrm{s}$. Curves acquired in the dark.
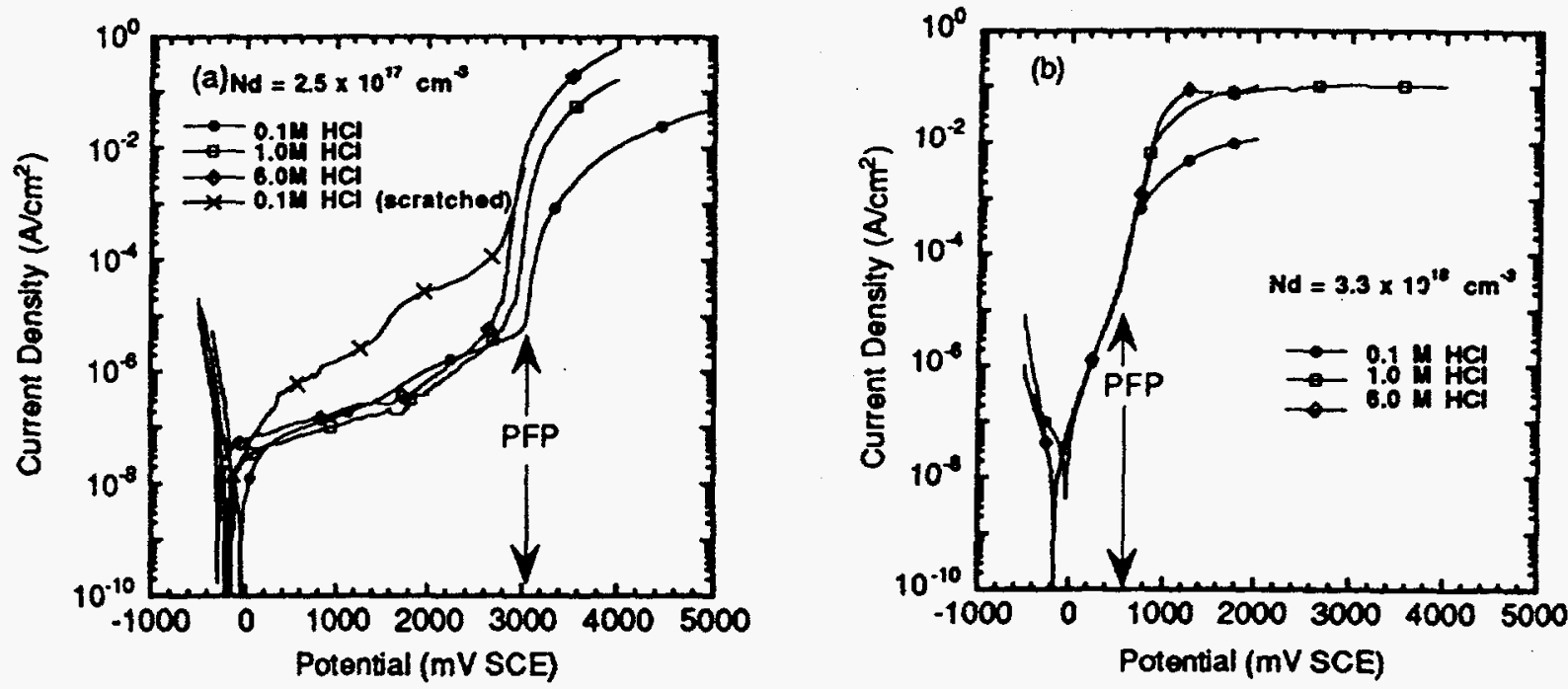

Fig. 2: Polarization curves of n-GaAs (100): a) doped with $2.5 \cdot 10^{17} \mathrm{~cm}^{-3} \mathrm{Si}$; b) doped with $3.3 \cdot 10^{18} \mathrm{~cm}^{-3} \mathrm{Si}$, in different $\mathrm{HCl}$ concentrations. Also included in (a) are the results of a sample which was lightly scratched with a diamond scribe prior to the measurement. The pore formation potentials (PFP) are indicated. Sweep rate $5 \mathrm{mV} / \mathrm{s}$. Curves acquired in the dark.

carriers that overcome the barrier by thermal activation. Therefore the current limiting factor in the electrochemical cell is the space charge layer in the GaAs. This current limitation by the space charge layer leads to a very similar polarization behaviour in the two electrolytes. Space charge layer control (diode behaviour) is also indicated by the absence of an active/passive transition in the polarization curves. 
In order to oxidize and dissolve $\mathrm{GaAs}$ according to $\mathrm{GaAs}+6 \mathrm{~h}^{+}+\mathrm{Ga}^{3+}+\mathrm{As}^{3+}, \mathrm{a}$ sufficient concentration of holes $\left(\mathrm{h}^{+}\right)$has to be provided at the surface. A considerable current flow under dark conditions is only possible if breakdown of the Schottky barrier occurs.

There are basically two mechanisms which induce the Schottky barrier breakdown. One is avalanche breakdown and the other is tunneling. Which process dominates is determined by the width of the space charge region. Apart from other semiconductor parameters, the width of the layer depends strongly on the doping concentration. For highly doped material the width of the layer can become thin enough that tunneling dominates whereas for lower doped material an avalanche breakdown mechanism is favored.

According to literature data [9] avalanche breakdown is expected to occur for the lower doped material at approximately $7 \mathrm{~V}$. However, an estimate yields that, even for the lower doped sample, tunneling contributions should be considered. This and the fact that the value of $7 \mathrm{~V}$ is based on calculations means that the avalanche or a mixed avalanche tunneling breakdown mechanism represents a relatively good explanation for the PFP of approximately 3 to $4 \mathrm{~V}$ obtained from the polarization curves.

If a pure tunneling breakdown is considered, for the higher doped sample, a breakdown voltage of approximately $0.8 \mathrm{~V}$ is expected [9], which is in excellent agreement with the PFP obtained from the polarization curves.

Further, it is well known that breakdown of the space charge layer is facilitated at surface defects, and this can explain the significantly higher currents in the case of the scratched sample.

\section{Pit Morphology and Pore Growth}

Figure 3 shows a series of SEM micrographs illustrating the evolution of the porous structure on n-type GaAs. In Fig. 3a the sample was removed from the electrolyte as soon as a current increase was detected and hence represents a very early stage of the attack. Under these conditions only a few pits can be observed on the surface. These pits indicate that initially crystallographic etching occurs, exposing the $(111\}$ planes of the material (it is also interesting to note that a certain degree of undercutting occurs). From these initial etch pits the attack spreads laterally leaving distinct units behind, as shown in Figs $3 b, c$ for a sample exposed to conditions slightly more anodic than that shown in Fig 3a. Exposure of the sample for longer times above the PFP leads to complete conversion of the surface to a porous structure.

Figure 3d shows an SEM image of a porous region on a sample exposed to $0.1 \mathrm{M} \mathrm{HCl}$ at $6 \mathrm{~V}$ SCE for 5 min, i.e., well above the PFP. A cross-sectional SEM image (Fig. 3e) reveals a needlelike appearance. Typically these needles are topped with a layer of crystallites. Higher magnification of the needle-like structure (Fig. 3f) yields a representative feature size in the micrometer to sub-micrometer range. A higher magnification of the top layer on samples prepared under different conditions (Fig 3g,h) shows within this layer the presence of significantly lower feature sizes.

From the SEM results, it is evident that a preferential attack perpendicular to the (100) direction takes place, i.e., along $\langle 011>$. Angle measurements of the remnant needle-like structure are in quite good agreement with $\{111$ \} planes being exposed. This is consistent with the fact that [111] planes usually have the lowest reactivity with respect to chemical dissolution [10-12].

This seems the most likely reason for the preferential attack leading to the formation of the porous structure. This process can nevertheless only take place when, within a pore, activation (kinetic) control of dissolution is established, since diffusion control would lead to a uniform etch behavior. 


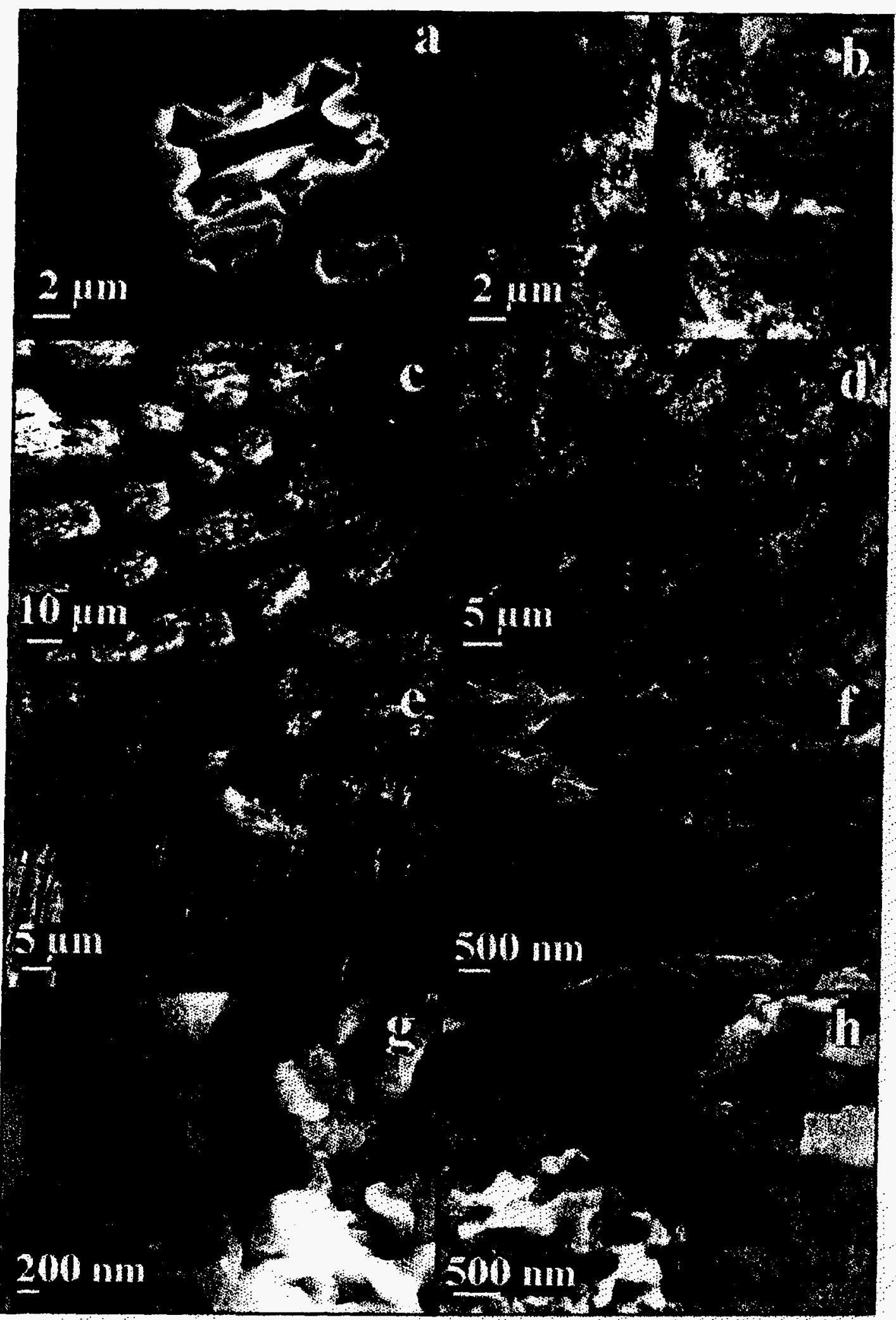

Fig. 3: SEM micrographs of $n-G a A s ~(100)$ after polarization in $0.1 \mathrm{M} \mathrm{HCl}$ :

a) sample removed from electrolyte as soon as a current increase was detected (i.e., at the PFP) b,c) different locations on a sample after polarization for $5 \mathrm{~min}$ at $3 \mathrm{~V} \mathrm{SCE;} \mathrm{d)} \mathrm{attacked} \mathrm{patch} \mathrm{on} \mathrm{a}$ sample after polarization for $5 \mathrm{~min}$ at $6 \mathrm{~V}$ SCE ; e) cross-section of Fig. 3d ; f) higher magnification of a lower needle like structure of Fig. 3d. ; g,h) small porosity apparent on surface locations on porous GaAs formed by $\mathrm{g}$ ) a potential sweep from $-0.5 \mathrm{~V}$ SCE to $6 \mathrm{~V}$ SCE in $0.1 \mathrm{M}$ $\mathrm{HCl}$; h) a potential step (8V SCE, $5 \mathrm{~min})$ in $0.1 \mathrm{M} \mathrm{HCl}$. 
In order to obtain information on the composition of the porous structure, AES sputter profiles were acquired on a heavily attacked region of the sample shown in Fig. 3d and compared with an AES profile of a standard, clean GaAs sample (Fig. 4). It is clear that most of the porous structure shows a Ga:As ratio close to 1:1, except for a region near the outer surface. In this region an enrichment of $\mathrm{As}$ is observed which coincides with an increased $\mathrm{Cl}$ concentration in the same region.

Similar results are obtained from an EDX investigation of features seen in Fig. 3e. If the EDX analysis is performed on the top crystallite structure, a As/Ga ratio typically in the range of 2:1 is obtained, whereas an analysis of the lower needle-like structure results in a ratio close to 1:1.

The fact that the underlying porous structure consists of stoichiometric GaAs is in good agreement with work by Tromans et al. [5] who found that $\mathrm{GaAs}$ dissolves in acidic solutions as GaAs. Thus no selective dissolution of one of the components takes place during pitting corrosion and the remaining structure is also expected to consist mainly of GaAs.

XPS investigations were performed in order to elucidate the origin and the role of the chloride ions. In the case of a similar phenomenon, the lacalized dissolution of metals in chloride containing solutions, often a specific adsorption of chlorides or at least a significant enrichment of chloride on the surface prior to the attack is assumed to represent a precursor state of the attack. Samples were polarized below and above the PFP in $0.3 \mathrm{M} \mathrm{NH}_{4} \mathrm{H}_{2} \mathrm{PO}_{4}$ in the presence and absence of chlorides and XPS spectra acquired. Figure 5 shows the $\mathrm{Cl} 2 \mathrm{p}$ and $\mathrm{P} 2 \mathrm{~s}$ regions, i.e., for both anions present in the electrolyte. From the results it is clear that chloride can only be detected on the sample surface once pore formation has been initiated and incorporation of chlorine in the surface film occurs simultaneously with a detectable uptake of phosphorous. Thus it is very likely that this anion uptake is simply due to the migration of electrolyte anions into the pores to compensate for the high cation $\left(\mathrm{As}^{3+}, \mathrm{Ga}^{3+}\right.$ ) flux out of the pores.

$A$ more detailed analysis of the results shows the presence of oxides $\left(\mathrm{Ga}_{2} \mathrm{O}_{3}\right.$ and $\left.\mathrm{As}_{2} \mathrm{O}_{3}\right)$ as well as chlorides (mostly As bound) and phosphate (mostly Ga bound).

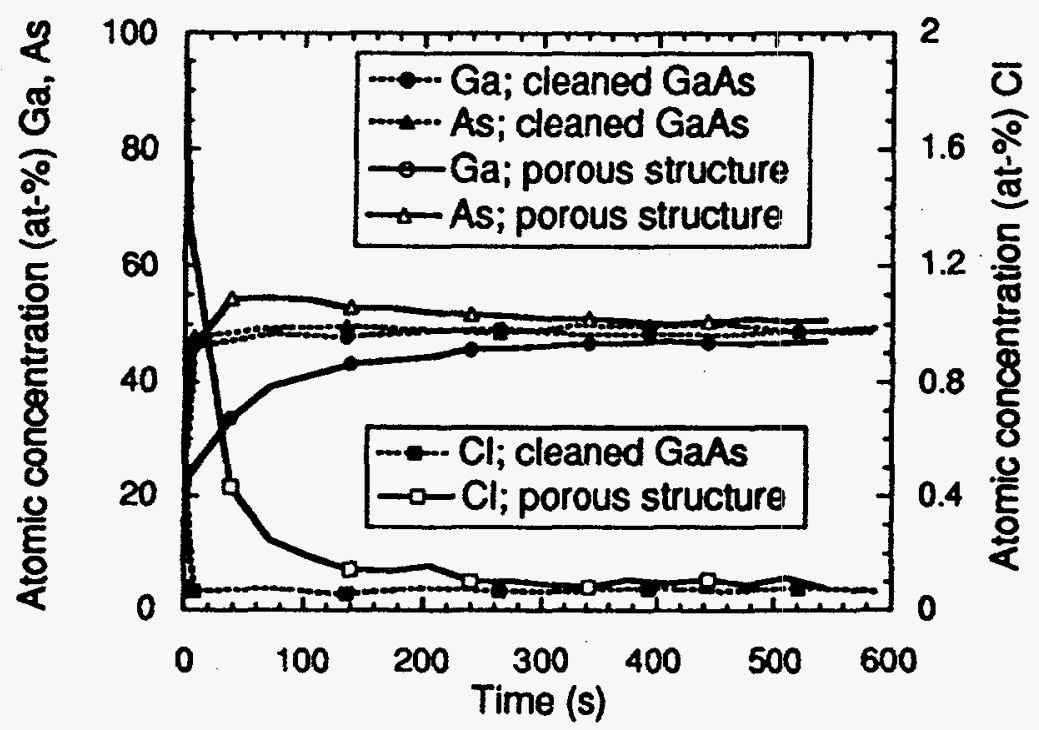

Fig. 4: AES sputter profiles of the porous structure of Fig. 3d and a reference sample of standard, clean GaAs (100). Sputtering was by $1 \mathrm{keV}$ Argon ions. 


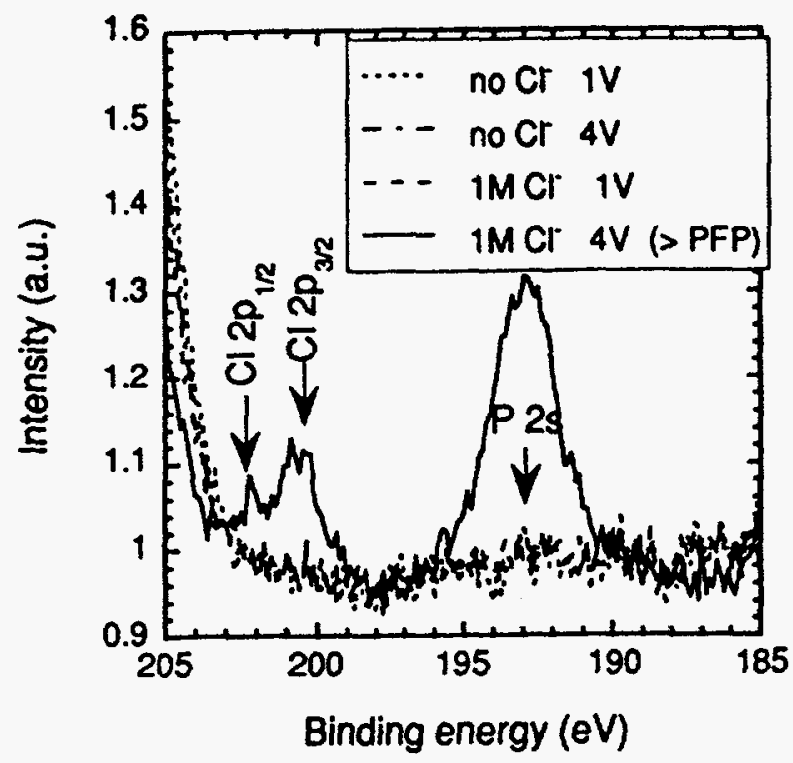

Fig. 5: XPS spectra showing the $\mathrm{P} 2 \mathrm{~s}$ and the $\mathrm{Cl} 2 \mathrm{p}$ region for n-type $\mathrm{GaAs}(100)$ polarized in $0.3 \mathrm{M} \mathrm{NH} \mathrm{NH}_{2} \mathrm{PO}_{4}$ with and without the addition of chlorides and below and above the pore formation potential (PFP).

\section{PL Properties of the Porous Structures}

Preliminary results from porous $\mathrm{GaAs}$ structures show that a significantly different PL spectrum can be obtained compared with bulk GaAs. A typical example is shown in Fig. 6. For the porous sample the maximum in the infrared emission is shifted from $860 \mathrm{~nm}$ (clean GaAs) to $840 \mathrm{~nm}$. In addition to the "infrared" peak at $840 \mathrm{~nm}$ a "green" contribution at $540 \mathrm{~nm}$ occurs. This "green" light emission can, under certain conditions, be intense enough to be visible to the naked eye and varies in lineshape and peak wavelength from sample to sample [13].

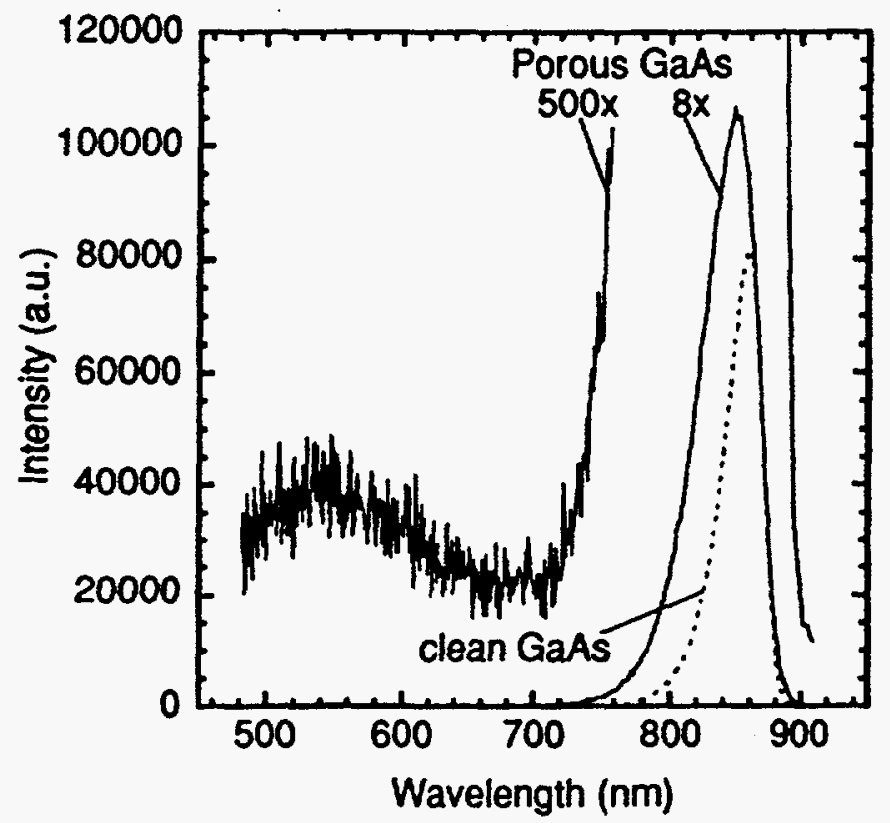

Fig. 6: Room temperature PL spectrum from the sample shown in Fig. 3g and from a clean n-type GaAs (100) surface. 
Porous $\mathrm{GaAs}$ can be grown anodically in $\mathrm{Cl}^{-}$-containing solutions above a certain threshold potential, the pore formation potential (PFP). The earliest stage of the localized dissolution process is the formation of crystallographic etch pits on the surface from which, under more severe conditions, the attack spreads laterally. Etch pit formation and the geometry of the porous structure are mainly influenced by the lower reactivity of the $[111\}$ crystal planes.

The PFP of n-type GaAs strongly depends on the doping level and the presence of surface defects. The value of the PFP can be correlated with the breakdown voltage of the space charge layer at the n-type GaAs/electrolyte interface.

The porous structure consists predominantly of stoichiometric $\mathrm{GaAs}$ and contains oxide and chloride compounds of $\mathrm{Ga}$ and As. Chloride uptake is only detected after the pore formation process has been initiated; a precursor state involving $C l$ was not found.

Preliminary investigations show visible and infrared photoluminescence from porous GaAs due to quantum confinement effects.

\section{ACKNOWLEDGMENTS}

The authors would like to thank John Phillips (NRC) for performing the Auger depth profiles and H.J. Labbe (NRC) for technical assistance in the PL measurements. One of the authors (PS) would like to thank the Swiss National Science Foundation (NF) for financial support during his stay at NRC. This work was performed in part under the auspices of the U.S. Department of Energy, Division of Materials Sciences, Office of Basic Energy Science under Contract No. DEAC02-76CH00016.

\section{REFERENCES}

1 Whole edition of J. of Luminescence, 57, (1993).

2 S. M. Prokes, Interface, 3, 41 (1994).

3 N. G. Ferreira, D. Soltz, F. Decker, and L. Cescato, J. Electrochem. Soc., 142, 1348 (1995).

4 B. H. Erne, D. Vanmeakelbergh, and J. J. Kelly, Advanced Materials, 7, 739 (1995).

5 D. Tromans, G. G. Liu, and F. Weinberg, Corros. Sci., 35, 117 (1993).

6 P. Schmuki, J. Fraser, C. M. Vitus, M. J. Graham, and H. Isaacs, J. Electrochem. Soc., (submitted) .

7 P. Schmuki, J. W. Fraser, C. M. Vitus, M. J. Graham, and H. S. Isaacs, Proc. of the Symposium "Critical Factors in Localized Corrosion II", Ed. P.M. Natishan, R.G. Kelly, G.S. Frankel, R.C. Newman, The Electrochemical Society, Proc. Vol 95-15, p. 226 (1995).

8 P. Schmuki, G. I. Sproule, J. A. Bardwell, Z. H. Lu, and M. J. Graham, J. Appl. Physics, (in press).

9 S. M. Sze, Physics of Semiconductor Devices, John Wiley \& Sons, New York, (1981).

10 H. C. Gatos and M. C. Lavine, J. Electrochem. Soc., 107, 472 (1960).

11 H. Seidel, A. Csepregi, A. Heuberger, and H. Baumgaertel, J. Electrochem. Soc., 137, 3612 (1990). 
12 B. Tuck and A. J. Baker, J. Mater. Sci., 8, 1549 (1973).

13 P. Schmuki, D. J. Lockwood, H. J. Labbe, and J. W. Fraser, Appl. Phys. Lett., (submitted for publication).

\section{DISCLAIMER}

This report was prepared as an account of work sponsored by an agency of the United States Government. Neither the United States Government nor any agency thereof, nor any of their employees, makes any warranty, express or implied, or assumes any legal liability or responsibility for the accuracy, completeness, or usefulness of any information, apparatus, product, or process disclosed, or represents that its use would not infringe privately owned rights. Reference herein to any specific commercial product, process, or service by trade name, trademark, manufacturer, or otherwise does not necessarily constitute or imply its endorsement, recommendation, or favoring by the United States Government or any agency thereof. The views and opinions of authors expressed herein do not necessarily state or reflect those of the United States Government or any agency thereof. 